\title{
Norois
}

Environnement, aménagement, société

\section{Technopôle et gouvernance publique : le cas de Sophia-Antipolis}

Technopôle and public governance: the case of Sophia-Antipolis

\section{Alexandre Grondeau}

\section{(2) OpenEdition}

\section{Journals}

\section{Édition électronique}

URL : http://journals.openedition.org/norois/1798

DOI : $10.4000 /$ norois. 1798

ISBN : 978-2-7535-1548-2

ISSN : $1760-8546$

Éditeur

Presses universitaires de Rennes

Édition imprimée

Date de publication : 1 septembre 2006

Pagination : $39-50$

ISBN : 978-2-7535-0342-7

ISSN : 0029-182X

\section{Référence électronique}

Alexandre Grondeau, «Technopôle et gouvernance publique : le cas de Sophia-Antipolis », Norois [En ligne], 200 | 2006/3, mis en ligne le 12 décembre 2008, consulté le 19 avril 2019. URL : http:// journals.openedition.org/norois/1798; DOI : 10.4000/norois.1798 


\title{
TeChNOpÔLE ET GouVERnANCE PUbliQue : \\ LE CAS DE SOPHIA-ANTIPOLIS
}

\author{
ALEXANDRE GRONDEAU \\ Laboratoire de Géographie urbaine - Université Paris 10 \\ barnabax@yahoo.fr
}

\begin{abstract}
RÉSUMÉ
Sophia-Antipolis est un des technopôles européens les plus dynamiques. Cet article a comme objectif d'analyser le rôle des acteurs publics dans l'émergence et le développement du technopôle et de voir quel rôle ils jouent aujourd'hui. Nous présenterons dans un premier temps les ambitions qui lui ont donné le jour et qui en ont fait un succès. Il s'agira également de montrer à travers un bilan, les atouts et les carences actuelles du parc. Nous verrons dans un second temps l'impact de la loi de renforcement et de simplification intercommunale dite "Chevènement " sur l'articulation et la coordination des acteurs locaux publics. Nous poserons la question de l'apport de cette loi pour le technopôle et nous analyserons le projet de territoire qui en découle.
\end{abstract}

MotS CLÉS : Gouvernance-Haute technologie - Loi « Chevènement »-Intercommunalité - Sophia-Antipolis - Technopôle - Technopole.

\section{ABSTRACT}

\section{Technopôle and public governance: the case of Sophia-Antipolis}

Sophia-Antipolis is one of the most dynamic technopole of Europe. This article aims at analysing the role of public actors in the emergence and development of the technopole and the role they play nowadays. We will first present the ambitions of Sophia-Antipolis at its beginning, which made it successful. We'll also show, through an assessment, the assets and the deficiencies of the park. We will present in a second time the impact of the so called "Loi de renforcement et de simplification intercommunale" on local public actors' articulation and coordination. We will analyse what the provisions of this Law brought to the technopole and the territorial project it entails.

KEY WORDS : High Technology - Public Governance - Research E Development - Science Park - Sophia-Antipolis - Technopole.

Plus de trente-cinq ans après sa mise en place, Sophia-Antipolis apparaît comme un technopôle dynamique et reconnu à l'échelle européenne et internationale. Cette réussite s'est appuyée, dans un premier temps, sur l'action des pouvoirs publics qui s'est notamment matérialisée par une politique d'aménagement issue du fonctionnalisme urbain (Le Corbusier, 1943). Au fil du temps, 
ALEXANDRE GrondEAU

ce modèle a montré ses limites en particulier en termes d'inflation foncière et de congestion des transports et appelle à de nouvelles réflexions sur l'avenir des technopôles (Grondeau, 2004). À l'heure de la globalisation et de la concurrence exacerbée entre régions, les acteurs institutionnels semblent avoir à jouer un rôle déterminant (Scott, 1999; Camagni et Maillat, 2006). L'ambition de cet article est de faire un bilan de l'action des acteurs publics de Sophia-Antipolis et de montrer comment la création d'un nouvel échelon territorial, ici la communauté d'agglomération, qui avait pour ambition d'optimiser le fonctionnement d'un territoire, peut perturber un technopôle et son mode de gouvernance déjà imparfait.

Nous analyserons dans un premier temps les modalités et les mécanismes de formation et de développement du technopôle pour saisir le rôle des acteurs publics. Nous ferons ensuite un bilan de Sophia-Antipolis et interrogerons ainsi le projet de départ à la lumière de la réalité actuelle. L'idée initiale de son fondateur Pierre Laffitte était de créer une «ville de la science et de la sagesse ». La ville n'a jamais émergé et Sophia-Antipolis est resté un technopôle, au sens strict du terme (Benko, 1991; Wackerman, 1992; Fache, 2002), qui accueille néanmoins plus de mille entreprises de haute technologie. Ce succès s'accompagne de problèmes inhérents aux processus d'agglomération urbaine : congestion et élévation des prix du foncier en tête, que les acteurs publics ont du mal à gérer. À partir de ces réflexions, il s'agit de faire un bilan de Sophia-Antipolis et de montrer les atouts et les faiblesses du technopôle.

Le deuxième axe de cet article analysera l'impact de la loi de simplification intercommunale de 1999 dite "Chevènement ». Nous verrons si cette loi a permis d'améliorer la gouvernance du technopôle. Si l'ambition nationale était de renforcer et de simplifier la complexité des échelons territoriaux, nous montrerons que la loi a ajouté de la confusion et de l'opacité au fonctionnement du technopôle entraînant un gel de près de deux ans des projets de développement. Le refus de Mougins d'intégrer la communauté d'agglomération nouvellement créée, et la remise en cause de la place du département dans la gestion du parc ont illustré les difficultés de "gouverner » Sophia-Antipolis. Nous verrons que malgré cela, une réflexion s'est amorcée quant au devenir du parc. Le projet de territoire vise à dépasser les carences actuelles en intégrant les principes de développement durable et les enjeux économiques actuels et à venir. Nous poserons la question de la pertinence territoriale de cette nouvelle échelle quant à ce projet et ses modalités d'actions.

\section{Sophia-Antipolis, historique et bilan}

\section{UNE CRÉATION PAR LES ACTEURS PUBLICS}

Sophia-Antipolis qui n'était alors qu'au stade d'idée, fut pensé par son instigateur, Pierre Laffitte, comme une ville de la science et de la sagesse. La volonté de ce dernier était alors claire : « délocaliser le quartier latin aux champs ». Ce slogan séduit les décideurs de l'époque puisque Laffitte, alors directeur de l'École des Mines, bénéficia de l'aide des organismes nationaux et locaux. C'est grâce au soutien du ministre Jean-Marcel Jeanneney, de la délégation à l'aménagement du territoire et à l'action régionale (DATAR) et des acteurs publics locaux tels que le Département, la chambre de Commerce et d'Industrie (CCI) des Alpes-Maritimes, des communes de Valbonne, d'Antibes, de Biot, de Mougins et de Vallauris (rejointes plus tard par la Colle-sur-Loup, Opio, Roquefort-les-Pins et Villeneuve-Loubet) alors réunies en syndicat mixte, que le parc d'activités de Sophia-Antipolis allait voir le jour. Après une première tentative avortée de gestion du technopôle par une structure privée, ce sont les acteurs publics qui ont porté l'émergence et le développement de Sophia-Antipolis, participant à la mise en place d'une intercommunalité de fait. Leur action a été double. Elle a d'abord porté sur l'organisation urbaine du technopôle et ensuite sur sa promotion.

Les acteurs publics ont amené avec eux leur savoir-faire et leur vision urbaine. Celle-ci s’inscrit à l'époque dans la filiation de la Charte d'Athènes (Le Corbusier, 1943) et du fonctionnalisme 
urbain. Il s'agit d'attribuer à chaque fonction de la ville (habiter, travailler, se récréer et circuler) un espace. La pratique du «zoning » fait partie intégrante de la politique de construction et du logement de l'État français (Chamboredon et Lemaire, 1970) et Sophia-Antipolis en est l'illustration. C'est une «ville fonctionnelle » qui a été initiée, pas une ville au sens classique du terme qui appelle différents éléments dont une histoire (un plan, une trame), un paysage caractéristique et une agglomération de la population et des activités. Ainsi l'INSEE parle de ville à partir de 2000 habitants agglomérés. Au moment de sa création, Sophia-Antipolis, ne réunit aucun des éléments que nous venons de citer et notamment pas la concentration urbaine. Le technopôle a été pensé comme une zone urbaine aérée et diffuse. Dès son lancement, le syndicat mixte qui gère Sophia-Antipolis (SYMISA) a comme ambition de maîtriser son développement en créant des zones de bureaux et des zones résidentielles, tout en maintenant une proportion d'espaces verts importante grâce à des prescriptions urbanistiques rigides de 2/3 d'espaces verts pour 1/3 de bâti. Le sommet des collines est protégé par une interdiction d'y construire. Les 1500 hectares d'espaces verts ont été rapidement aménagés en parcs départementaux et largement ouverts au public. Les axes routiers principaux vont délimiter le parc d'activité. D’abord, l'A8 orientée estouest qui constitue la frontière sud du parc. Ensuite, la route du parc, orientée nord-sud qui relie l'A8 à Valbonne, dont le tracé sinueux avait été conçu pour réduire la vitesse des automobiles, laisse à l'ouest la forêt et les espaces verts, et à l'est les principaux établissements de haute technologie (fig. 1).

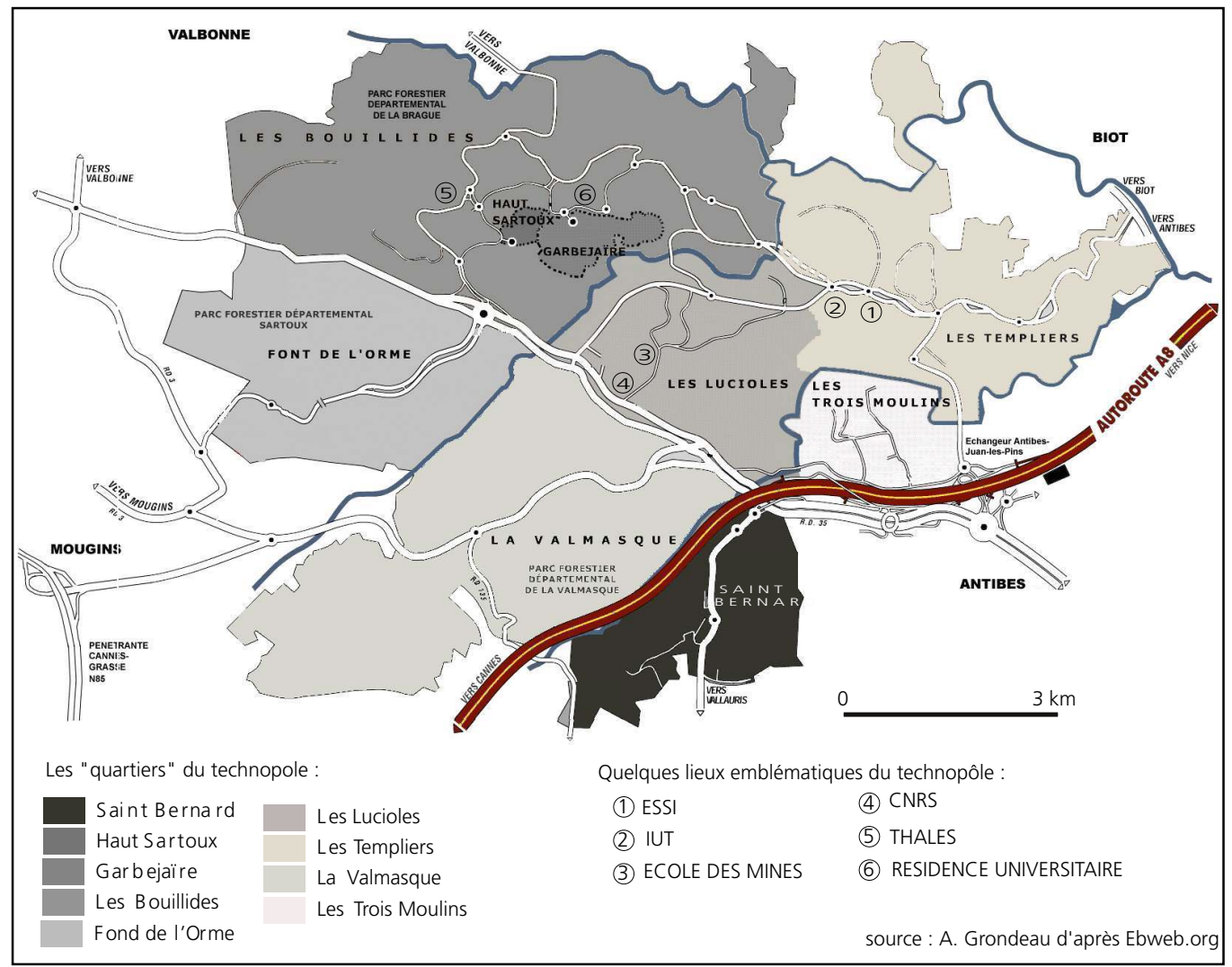

Figure 1 : Localisation du technopôle de Sophia-Antipolis (source : [Ebweb.org])

Location of the technopole of Sophia-Antipolis 
ALEXANDRE GRONDEAU

Ces deux éléments (transport et aménagement) ont contribué à la diffusion de l'urbanisation et à sa faible densité. Le réseau routier en arborescence contribue à organiser les bâtiments en grappes et limite les contacts entre ces groupes de bâtiments. La mixité habitat/activité est quasi nulle et va à l'encontre des projets initiaux de développement et d'animation urbaine de Sophia-Antipolis. On est plus proche de l'urbain ruralisé et du rural urbanisé théorisé et décrit par I. Cerda (1979) et E. Howard (1969) que de la ville au sens classique du terme contée par L. Mumford (1964).

L'émergence de Sophia-Antipolis est donc essentiellement due aux acteurs publics. Ils vont donner à ce territoire le potentiel urbain pour devenir un technopôle. Le succès de cette première phase se matérialise par l'implantation de grands groupes tels que IBM ou Texas Instrument qui vont être attirés par l'effet combiné de ce potentiel et par l'« héliotropisme et le renom de la Côte d'Azur » (Querré, 2002). Car au-delà de la production urbaine, les acteurs publics vont participer largement à la promotion de Sophia-Antipolis.

La communication urbaine a en effet pris une place importante dans l'attractivité du technopôle à ses débuts. Par ce terme on entend la définition d'un produit urbain dans l'optique de marketing et d'attraction d'entreprises, synonyme d'emplois et de taxe professionnelle. Tout un nouveau vocabulaire est employé afin de marquer la modernité des villes : compétitives, entreprenantes, ouvertes, «high-tech». Ainsi, on a entendu parler de Metz la « communicante », Montpellier la «surdouée » ou encore de Brest et de son « être à la pointe et le prouver». Les brochures de présentation de ces territoires permettent une argumentation qualitative autour du lieu d'accueil. Lyon, Grenoble, Toulouse ou Montpellier se présentent (à coup de campagnes publicitaires très importantes) comme des villes de l'intelligence et de l'invention. Sophia-Antipolis n'échappa pas à la règle et fut présenté comme idéal en termes de climat, de qualité du site et de sites alentour, de gastronomie, d'activités sportives (présence de golf, de tennis), d'un patrimoine architectural prestigieux, d'un urbanisme spectaculaire, de manifestations culturelles importantes... Tous les éléments susceptibles de contribuer à inscrire le mode de vie sous le signe de la modernité furent et sont encore invoqués. L'objectif était de vendre un cadre de vie idéal pour la vie des cadres et des entreprises de haute technologie.

C'est par ces deux types d'action publique axés sur la réalisation d'infrastructures urbaines et la promotion du site que le succès de Sophia-Antipolis fut initié. Il ne fut pas total comme nous allons le voir avec le bilan actuel du technopôle.

\section{UN BILAN MITIGÉ}

Nous allons présenter ce bilan en montrant que la réussite a engendré des problèmes récurrents que les pouvoirs publics ont du mal à surmonter. Nous nous basons pour ce faire, sur de nombreuses études lancées, en particulier par la préfecture, qui souhaitait avoir une connaissance approfondie de ce qu'elle appelait bassin de vie (en relation notamment avec les déplacements domicile - travail). Le diagnostic d'agglomération réalisé par la DDE était l'un de ces documents. Nous y avons été associé en tant qu’observateur. Il a mis un certain nombre d'éléments en lumière sur la réalité même des activités de haute technologie de l'agglomération. Nous allons les présenter rapidement et y avons ajouté les résultats d'études complémentaires que nous avons menées (Grondeau, 2000, 2001, 2003).

D'un point de vue économique, Sophia-Antipolis continue de grandir puisqu'il compte chaque année un nombre plus important d'entreprises de haute technologie. Ainsi, le SYMISA recensait 1227 raisons sociales en 2003 (soit 83 de plus qu'en 1999), et 24550 emplois (4 020 emplois de plus qu'en 1999). Si on y regarde de plus près, on peut arguer que la définition de " haute technologie » est prise de manière large (Fache, 1999), et qu'une des particularités du parc est de compter parmi elles de nombreuses grandes entreprises comme Amadeus (plus de mille emplois) ou Air France. Le poids important de ces dernières dans la structure générale de l'emploi de haute technologie la différencie de la Silicon Valley (Saxenian, 1994 ; Grondeau, 2005), paradis des start- 
up et autres petites et moyennes entreprises. À ce titre, on peut poser la question de l'ancrage territorial des entreprises du technopôle. J.-L. Gaffard (1993) estime que le territoire n'a rien à voir dans la stratégie de localisation d'une entreprise de haute technologie si rien n'est fait pour créer et pérenniser des relations et/ou des coopérations entre entreprises. À ce titre, il juge SophiaAntipolis déficitaire. Pour lui, les implantations sur le technopôle sont « fortement instables » dans la mesure où elles sont plus tournées vers l'extérieur (l'international) que vers l'intérieur (le local). M. Querré (2002) va dans ce sens quand il différencie une première phase du développement de Sophia-Antipolis tournée vers l'extérieur dans la mesure où elle se faisait principalement à partir de grands groupes, et une seconde phase récente plus endogène basée sur l'apparition de très petites entreprises et de quelques PME qui participent fortement à l'apparition d'un marché local. L'ensemble de ces entreprises maintient toutefois un taux de création supérieur à celui de la diminution des emplois. Les secteurs les plus porteurs du parc sont les sciences de l'information avec 319 raisons sociales pour 12131 emplois (soit une augmentation de 22 raisons sociales et 3133 emplois entre 1999 et 2003), et les sciences de la santé et de la vie avec 44 raisons sociales et 1947 emplois. Toujours selon M. Querré, les entreprises marquées «science de l'information » ont atteint un seuil qui permet l'apparition d'un milieu local dynamique et porteur (avec des TPE et PME) alors que les «sciences de la santé et de la vie » reste dans une configuration basique de laboratoires de grands groupes peu ancrés territorialement. Selon les projections établies au moment du diagnostic, le parc actuel offre une capacité résiduelle totale d'environ 10000 emplois soit une « réserve » évaluée, selon le rythme moyen constaté, à une dizaine d'années environ. On note malgré tout que la conjoncture économique peut fortement perturber le marché du travail, comme cela a été le cas après le crack du Nasdaq. Malgré ce potentiel et ces incertitudes, les acteurs publics agissent peu ou pas pour densifier les réseaux existants, renforcer l'articulation entre entreprises susceptibles de favoriser l'ancrage territorial. De ce point de vue, ils ne jouent par le rôle qu'ils devraient pour inscrire au mieux leur territoire dans la globalisation (Scott, 1999).

La population active du parc est une population jeune et qualifiée : $64 \%$ des actifs ont moins de quarante ans contre $46 \%$ pour le département. Le parc compte $52 \%$ de cadres, dont 17,5\% de cadres supérieurs. Cela confirme la qualification et la haute qualification de la force de travail caractéristique d'un technopôle (Benko, 1991 ; Fache, 2002). On note cependant que de nombreux cadres sont issus des entreprises venant s'installer. Cet élément concerne directement l'impact réel des entreprises sur le marché du travail local. J.-L. Gaffard précisait, en 1993, qu’à son avis Sophia-Antipolis n'était pas structuré par un «véritable marché du travail dans la mesure où une forte mobilité suscitée par le comportement des grandes entreprises n'est pas assortie d'un afflux significatif de ressources humaines ». Cela dit, le technopôle constitue un endroit à part dans le département puisque les revenus moyens sont supérieurs de $40 \%$ à la moyenne départementale. Mais là encore, les acteurs publics interviennent peu pour améliorer, orienter ou organiser les conditions de formation et de transparence du marché du travail qui est jugé opaque par les associations d'entrepreneurs.

L'université connaît une stabilisation après une période de développement qui a porté le nombre d'étudiants sur le parc à plus de 2500 . Ce chiffre est pourtant très faible, même si plus de 20000 étudiants sont présents à Nice, comparé à ce que d'autres technopôles comptent comme nombre d'étudiants. À titre de comparaison, Stanford sur la Silicon Valley accueille chaque année 14000 étudiants dont $50 \%$ en troisième cycle. L'État ne décentralise plus, ou moins, ses instituts de recherche publique comme il a pu le faire à la création du parc (École des Mines, INRIA, CNRS). Les carences du technopôle sont manifestes aussi bien en termes de nombre, qu'au niveau des relations Recherche - Industrie ou des collaborations entre les laboratoires publics et les structures privées qui ne sont pas flagrantes. Les acteurs locaux publics ne jouent pas ou peu le rôle de facilitateurs ou d'initiateurs de ces relations comme ils pourraient le faire en participant à la mise en place ou l'animation de plates-formes technopolitaines caractéristiques (incubateurs, associations, clubs). Ce sont les acteurs privés qui jouent ce rôle. 
ALEXANDRE GRONDEAU

La composante transport de Sophia-Antipolis est caractérisée par sa saturation aux heures de pointe. Ceci est inquiétant, car la fluidité des transports est un élément primordial dans la venue et l'installation d'une entreprise de haute technologie et de ses cadres. Ce genre de problèmes et leurs résolutions rentrent dans les exigences que la force de travail et les universitaires ont à l'endroit de leur lieu de travail. Cela est à mettre en parallèle avec les carences de l'habitat de SophiaAntipolis. Les prescriptions urbanistiques rigides et la densification de l'urbanisation improbable qui en découlent ont des conséquences importantes. Les parcs HLM, locatif et en accession sont inadaptés, en raison de l'inflation récurrente de l'immobilier. Les jeunes actifs, les étudiants, les habitants d'origine qui souhaitent évoluer dans leur " cursus » résidentiel, les ménages à niveau de vie modéré voire les cadres supérieurs ne peuvent plus se loger sur le technopôle. La doctrine du fonctionnalisme urbain a atteint ses limites.

Malgré ses carences, les études commandées par les acteurs publics ont conclu que les résultats de Sophia-Antipolis étaient bons dans l'ensemble, mais que le technopôle devait être à la hauteur des enjeux que posaient la saturation des transports, l'inflation du foncier, les relations entre la recherche publique et privée, et entre entreprises. On peut ajouter que les enjeux dégagés en 1999, mais récurrents depuis la fin des années 1980, demeurent sans que rien ne soit véritablement proposé pour y remédier. C'est ce que ne cessent de rappeler les clubs et associations d'entreprises critiquant les pouvoirs publics et leur lenteur à intervenir sur les problèmes du parc (notamment sur les problèmes de transport). Cette « gouvernance privée » évoquée par M. Querré (2002) joue un rôle fondamental depuis la fin des années 1980, car elle a pris le relais dans les mécanismes de développement du technopôle notamment grâce à l'activité de veille territoriale et de lobbying des associations d'entreprises, mais également par la création d'un espace de marché local. Elle illustre malgré tout les difficultés d'articulation que rencontrent acteurs institutionnels et acteurs économiques.

C'est dans ce contexte qu'une communauté d'agglomération a été instituée avec comme ambition de répondre aux carences du technopôle tout en développant ses atouts.

\section{Sophia-Antipolis à l'épreuve de la loi «Chevènement » et d'un projet d'agglomération}

\section{IMPACT DE LA LOI « CHEVÈNEMENT » SUR LE TECHNOPÔLE}

La loi no 99-586 du 12 juillet 1999, dite Chevènement, relative au renforcement et à la simplification de la coopération intercommunale a institué autour du technopôle, la communauté d'agglomération de Sophia-Antipolis (CASA). Son nom même montre à quel point le parc a valeur de moteur pour la zone. Le 7 septembre 2001, le préfet des Alpes-Maritimes, Jean-René Garnier, après de nombreuses consultations, prenait l'arrêté délimitant le périmètre de la CASA (fig. 2). Celle-ci a en charge quatre compétences obligatoires : le développement économique, l'aménagement de l'espace communautaire, l'équilibre social de l'habitat et la politique de la ville. Le technopôle semblait rentrer de plein droit dans la compétence obligatoire de développement économique, mais ce fut un sujet très sensible comme nous allons le voir. La CASA due également choisir trois compétences optionnelles parmi la voirie d'intérêt communautaire, l'assainissement, l'eau, la protection de l'environnement et les équipements culturels et sportifs.

L'application de la loi Chevènement a été mal perçue et difficilement vécue par certains acteurs publics locaux. En effet, se sont posées les questions de la mutualisation et de la répartition des richesses (taxe professionnelle en tête) et de la pertinence du fonctionnement du parc d'activité en dehors ou à l'intérieur du groupement intercommunal en création. La communauté d'agglomération validée par le préfet réunit quatorze communes. Parmi elles, huit des neuf communes fondatrices du technopôle ont accepté le projet d'agglomération. Seul Mougins a refusé d’intégrer le groupement intercommunal. La principale raison est politique. Historiquement parlant, la commune a toujours été plus tournée vers Cannes et ce, malgré la présence d’une partie du technopôle 
sur son territoire. Lorsque les délimitations des périmètres intercommunaux ont été réalisées, la commune a affiché son désir de se voir rattachée à une éventuelle communauté d'agglomération regroupant Cannes et Le Cannet. Cette situation a débouché sur un imbroglio administratif qui a été alimenté par la perte de pouvoir d'acteurs jusqu'alors incontournables et fédérateur dans le fonctionnement de Sophia-Antipolis (en particulier le Département).

Figure 2 : Le périmètre de la CASA (source : Fondation Sophia-Antipolis, 2002)

Area of la CASA

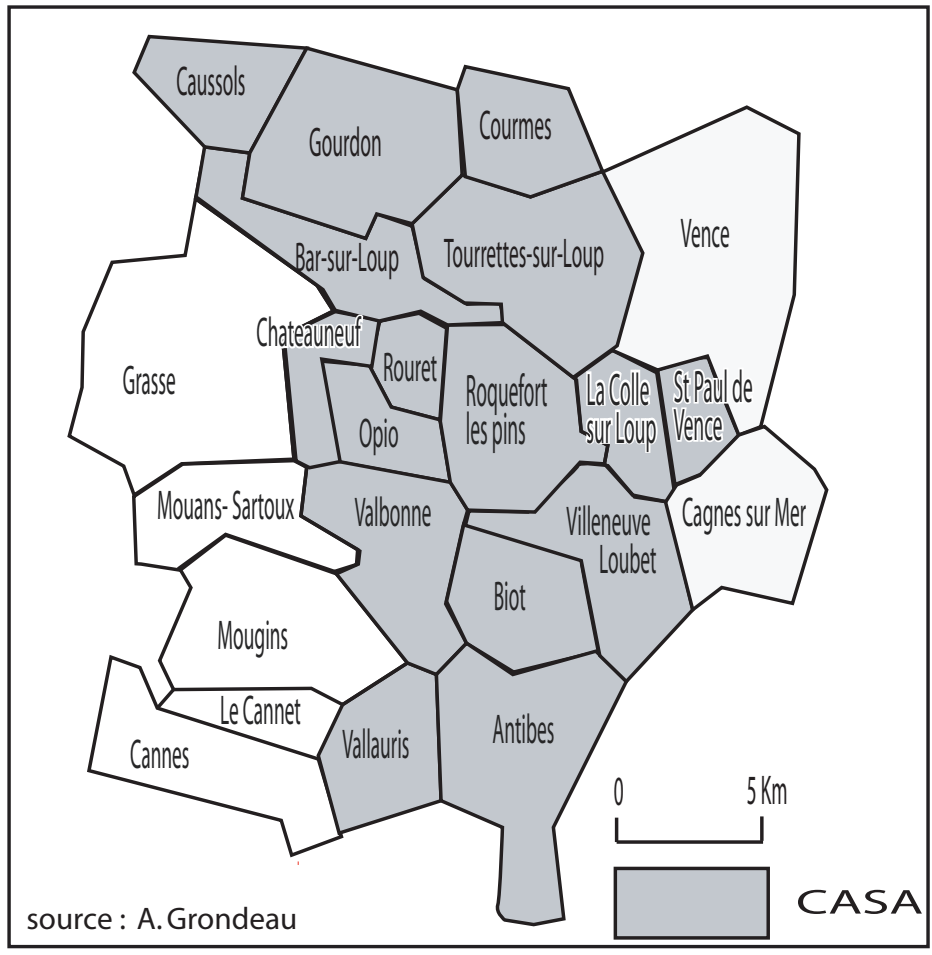

En ce qui concerne la place de Mougins au sein de Sophia-Antipolis, le rapport de force a été engagé par la commune qui a attaqué le statut de la CASA devant le tribunal administratif fin 2002. La commune refusait que la zone soit gérée par la CASA dont elle ne faisait pas partie. Le tribunal administratif a dans un premier temps donné raison à la commune et tort au préfet. Cette situation a permis au syndicat mixte en charge de Sophia-Antipolis, le SYMISA, (que la loi Chevènement avait mis sur la touche et auquel le département appartient) d'obtenir un mandat provisoire supplémentaire d'un an. Le jugement du tribunal administratif a donc stoppé le transfert de compétences, ce qui n’a pas contribué à rassurer les entreprises qui critiquaient déjà les instances politiques de la zone. La situation juridique restait très complexe. Suite à la décision du tribunal administratif, la CASA a fait appel et a demandé un pourvoi en cassation. Devant ce blocage, une réunion extraordinaire a été réunie par le préfet avec les représentants de l'État, du Conseil général, de la CASA, du Conseil régional, de l'Université et de CCI. Cette instance appelée «Comité Exécutif » s'est réunie régulièrement avec différents ordres du jour, pour ne pas laisser la situation en attente. Un consensus est sorti de ce groupe de travail. Finalement, le SYMISA resta gestionnaire du parc. Sa composition changeait au profit d'une redistribution des sièges. La CASA rentra avec $30 \%$ des sièges, au même niveau que le Conseil général. La Région obtint $20 \%$ des sièges, la CCI, $15 \%$ et Mougins $5 \%$. La CASA dispose néanmoins des moyens de faire-valoir ses arguments et de les mettre en application puisque, en plus de ses $30 \%$, elle dispose d'un certain nombre de sièges « amis » aux conseils général et régional. Parallèlement 
ALEXANDRE GRONDEAU

à ces problèmes administratifs et politiques, plusieurs questions sont encore à l'ordre du jour concernant le développement de Sophia-Antipolis et la pertinence du technopôle est remise en question par une nouvelle association, Initiative Riviera, qui réunit au niveau départemental les entreprises de haute technologie et qui souhaite une organisation globale, à son échelle, plutôt que limitée à un parc. Il s'agit de la réponse du Département à sa perte de pouvoir au sein du parc d'activités. D'autres éléments problématiques demeurent comme la scission non résolue de Mougins ou le désir de Cannes de développer son accueil des activités de haute technologie, en dehors de Sophia-Antipolis. Tout cela s'inscrit au moment même où Marseille et Aix essaient de développer un technopôle concurrent à Sophia-Antipolis.

La loi «Chevènement » a ainsi participé à une réorganisation et une redistribution des rôles des acteurs publics dans la gestion du technopôle. Le Département, qui a joué un rôle prépondérant depuis la création du technopôle et qui avait une place importante dans le SYMISA, s'est vu débarqué d'une grande partie de son pouvoir au détriment du groupement intercommunal et de sa commune leader, Antibes. En conséquence de quoi elle a développé, par le biais de son agence Initiative Riviéra, une nouvelle vision technopolitaine qui ne s'arrête plus à Sophia-Antipolis mais qui recouvre désormais tous les Alpes-Maritimes. Ces luttes de pouvoir illustrent la difficulté à " gouverner » Sophia-Antipolis qui n'avait pas besoin de cela au vu des enjeux dégagés par son bilan.

Cette situation n'a pas arrangé les relations entre acteurs publics et acteurs privés. La mise en place d'une communauté d'agglomération, censée simplifier les choses, n'a semble-t-il pas convaincu les entreprises présentes sur le parc, qui mettent en avant le manque de lisibilité et la lourdeur des institutions publiques. Le cas de l'harmonisation de la taxe professionnelle et la définition d'une taxe professionnelle unique (TPU) sont, à ce titre, exemplaires. Son instauration à $16,25 \%$, après de longues tractations, a une implication directe (une hausse) sur les entreprises présentes sur le parc, à l'exception de celles situées sur Antibes et Vallauris qui verront leur taxe professionnelle baisser de plusieurs points. Cet élément n'est pas à négliger dans la grogne de certains chefs d'entreprise, car les trois quarts des entreprises de Sophia-Antipolis verront leurs impôts augmenter. Valbonne qui accueille une grande partie des entreprises de haute technologie n'a pas vue cette hausse d'un bon œil même si elle a dû s'y soumettre.

La loi «Chevènement » n'a pas, depuis son lancement, permis une meilleure articulation des acteurs publics pour la gouvernance de Sophia-Antipolis. Son objectif de renforcer et de simplifier la coopération intercommunale a plutôt renforcé la complexité des relations entre acteurs publics participant à la gestion du technopôle. Pire, elle a ajouté de l'incertitude quant à l'avenir à court terme du technopôle, les projets de développement étant gelés ou suspendus. Elle n'a pas arrangé les relations entre acteurs publics et acteurs privés. Ceux-ci se sont trouvés confortés dans leurs critiques des acteurs publics. Et l'on peut se demander si l'intercommunalité vécue depuis plus de trente ans au sein d'un syndicat mixte n'était pas meilleure comparée à cette intercommunalité imposée par l'État. Cette nouvelle étape de la décentralisation n’a pas, pour le moment, trouvé une application pertinente en termes de gouvernance sur Sophia-Antipolis. Le "Comité Exécutif » a malgré tout cherché à réfléchir, sans pouvoir agir, sur le devenir de l'agglomération intégrant la plus grande partie du technopôle.

\section{LE PROJET D’AGGLOMÉRATION DE LA CASA}

\section{ET LA PERTINENCE D'UN NOUVEL ÉCHELON TERRITORIAL}

Pendant que la question du transfert des compétences de Sophia-Antipolis était discutée devant le tribunal administratif, les services techniques de la CASA étaient constitués et lançaient un certain nombre de réflexions sur les thématiques économiques, du transport, de l'habitat et de l'environnement. L'analyse de ces réflexions va nous permettre d'évaluer la pertinence de ce nouveau découpage territorial et de ses prérogatives. Nous avons pris le parti de distinguer deux axes d'intervention des acteurs publics sur la communauté d'agglomération. D'abord, tout ce qui à 
trait à l'urbain (infrastructures, cadre de vie) et qui correspond à leur rôle historique joué sur le technopôle. Ensuite, ce qui concerne le volet économique où les acteurs publics avaient montré des limites certaines quant à leur capacité d'encourager le développement de Sophia-Antipolis.

L'ambition affichée de la CASA est de dépasser les carences du technopôle grâce à sa nouvelle échelle d'intervention. Il ne s'agit plus d'agir sur un parc d'activités, mais sur une zone jugée plus pertinente à la résolution des problèmes rencontrés. La communauté d'agglomération pourrait permettre de dépasser certains freins notamment en ce qui concerne le cadre de vie. En effet, la plupart des carences et problèmes rencontrés par le technopôle se retrouve sur la communauté d'agglomération, en particulier l'absence de mixité logements - bureaux. La réalisation du SCOT doit permettre de réfléchir à la conservation de l'environnement et de ces espaces de qualité, en les protégeant et les valorisant. Le développement de l'habitat, l'amélioration des déplacements et la recherche de l'équilibre commercial sont également de sa compétence. La force du SCOT est qu'il porte sur la totalité du territoire de la CASA. Sa réalisation pose néanmoins la question de la pertinence de l'échelle territoriale. Toutes les communes voudront-elles jouer le jeu de la complémentarité quitte à accueillir de nouveaux logements dont certains seront forcément sociaux pour être en accord avec la loi Besson et son quota de $20 \%$ de logements sociaux? La réalisation d'un PLH, plan local d'habitat, doit en effet venir se greffer en parallèle sur le SCOT. Il devra rechercher un meilleur équilibre social puisque nous l'avons vu, le parc connaît de grosses carences de logements pour les étudiants et les catégories moins qualifiées. La CASA prévoit d'entamer une politique de réserve foncière qui devra satisfaire ces besoins. En aura-t-elle les moyens et sera-t-elle capable de dépasser le cadre des intérêts particuliers des communes? Autrement dit, l'intercommunalité de loi Chevènement est-elle plus opérationnelle que l'intercommunalité de fait qui existait depuis plus de trente ans? En intégrant le technopôle dans une réflexion plus globale portant sur l'agglomération, on peut craindre que la recherche de compromis entre communes soit une perte de temps qui se fasse au détriment de solutions pragmatiques ciblées et plus efficaces. C'est une des craintes des clubs et associations d'entrepreneurs du technopôle qui voient dans le nouvel échelon territorial une dilution de la capacité d'agir du SYMISA.

À ce titre il est bon d'évoquer le transfert de la compétence «protection et mise en valeur de l'environnement et du cadre de vie » à la communauté d'agglomération de Sophia-Antipolis. L'intercommunalité de projet et la mise en place d'une charte intercommunale de l'environnement doivent permettre de dépasser l'approche environnementale classique (solutions dégagées grâce aux documents d'urbanisme, ou des lois) pour instituer une véritable politique de développement durable basée sur la recherche de compromis. Cette charte prendra la forme de projets qui définiront leurs objectifs, leurs actions, leurs délais et financements. Sa gestion intégrera les associations, les acteurs privés et publics, afin d'avoir plus de chance de concrétiser les projets dégagés. Il s'agit ici d'instituer une véritable gouvernance en matière environnementale qui devra limiter une certaine forme d'immobilisme de l'aménagement du territoire. Mais comment concilier le besoin d'un grand nombre de logements nécessitant des opérations immobilières importantes et le respect d'un cadre de vie jugé intouchable par la population? La recherche de compromis pourra-t-elle aller jusqu'à la remise en question du cadre rigide des $2 / 3$ d'espaces verts pour $1 / 3$ d'urbain qui est un des éléments fondateurs du technopôle et de son organisation urbaine ? Une décision aussi forte peut-elle être le fruit d'un compromis entre acteurs? Rien n'est moins sûr. Penser l'aménagement de Sophia-Antipolis passe par le dépassement d'une contradiction de la population qui en tant que force de travail souhaite voir améliorer les capacités de développement économique du technopôle mais en tant que groupe de citoyens ne veut pas entendre parler de remise en question de certains espaces verts. La loi Chevènement ne résoudra pas ce problème par sa seule existence.

En ce qui concerne les transports, autre carence du parc et de l'agglomération, un accent tout particulier est porté sur la nécessité de développer les moyens de déplacement alternatifs. Le PDU, plan de déplacement urbain, doit permettre la mise en cohérence du réseau routier actuel et des projets de TCSP, et de créer des infrastructures adaptées (parkings de délestage, pistes cyclables, 
ALEXANDRE GRONDEAU

sentiers pédestres, etc.). Mais l'échelle intercommunale remet en question la zone qui était jusqu'alors prise en compte et qui allait de Cannes à Grasse en passant par Antibes, intégrant de fait le technopôle. Les carences ne seront pas résolues par un périmètre imposé mais par un périmètre pertinent. Là encore, le cadre national de la loi pose des problèmes qui peuvent s'apparenter à un retour en arrière concernant cette problématique.

C'est pourtant autour des questions de logement, de congestion et d'environnement que le SYMISA avait montré ses limites. Le nouvel échelon territorial, instauré par l'État, complique les données du problème en ajoutant un nouveau périmètre de réflexion pour lutter contre des carences qui nécessitent des actions ciblées. Il semblerait plus pertinent de définir des périmètres d'action, à géométrie variable, en fonction de chaque thématique et d'agir en fonction. L'État qui a joué un rôle moteur dans la création du technopôle devient un frein à son développement.

L'aspect économique est tout aussi inquiétant. Le projet de la communauté d'agglomération est d'arriver à ancrer « psychologiquement » Sophia-Antipolis au cœur du territoire de la CASA, et de réussir le mariage entre le passé et le futur, la culture provençale, méditerranéenne et le multiculturalisme, les métiers artistiques et technologiques, les activités de terroir et internationales. Pour ce faire, il faut améliorer les équipements de la zone afin d'assurer son développement, renforcer la recherche publique en attirant des organismes nationaux, développer certains pôles comme la "santé, les sciences de la terre et de l'environnement ", encourager le capital-risque à investir. Ces derniers éléments restent à l'état de bonnes intentions et on ne voit malheureusement pas grand-chose d'envisagé pour que ces carences évoluent. D’abord parce qu'ils dépendent d'autres échelons territoriaux, en particulier pour la localisation d'organisme de recherche public, que celui du groupement intercommunal. Ensuite parce que l'articulation entre acteurs publics et acteurs privés n'est pas satisfaisante et le projet global n'est pas convaincant.

D’un point de vue économique, la CASA souhaite s'appuyer sur le technopôle, mais également sur le tourisme et le tissu économique traditionnel. L'expérience accumulée sur le parc est envisagée comme devant servir aux secteurs traditionnels (métiers artistiques, manuels, agricoles). Le technopôle doit pouvoir diffuser ses innovations, notamment en ce qui concerne les nouvelles technologies de l'information et de la communication, au niveau de l'agglomération. La CASA intègre ainsi parfaitement le technopôle dans son projet d'évolution. Sophia-Antipolis, qui n'était qu'un parc d'activités dédié aux hautes technologies, est désormais envisagé comme une agglomération. Mais l'intégration d'une politique de développement économique ciblée bien qu'imparfaite dans une politique de développement plus générale va-t-elle satisfaire les entreprises de haute technologie qui étaient déjà très critiques?

La CASA justifie cette prise en compte des activités complémentaires par la nécessité de se protéger contre les aléas économiques qui ont un impact très fort sur la recherche et le développement et la haute technologie. Il s'agit, pour reprendre les termes d'un technicien de la CASA, « de ne pas mettre tous ses œufs dans le même panier ». Le tourisme a un rôle important à jouer puisqu'il s'agit, selon les études menées, d'une des deux activités principales de la CASA. Le patrimoine naturel, historique et culturel, les métiers traditionnels, l'artisanat encouragent l'agglomération à développer un tourisme scientifique, culturel, d'affaire. Un tourisme qualitatif par opposition au tourisme de masse reste donc le choix du groupement intercommunal. Ces activités restent pourtant largement dépendantes de la conjoncture économique au même titre que les activités de haute technologie.

Pour ce qui concerne l'emploi, le technopôle reste au cœur du projet. Un schéma de cohérence du développement économique doit débuter afin de mieux percevoir l'offre et la demande d'emplois. Une étude devrait être menée dans la foulée concernant la mise en place de formations adaptées aux besoins des entreprises de haute technologie présentes sur la CASA. Un des enjeux majeurs de la future gouvernance entre acteurs publics et acteurs privés est que l'avis des clubs et associations d'entreprises du technopôle soit entendu par le groupement intercommunal, qui dans l'état actuel des choses a perdu beaucoup de son crédit. Il faut ajouter que le bassin d'emploi 
de Sophia-Antipolis dépasse largement le périmètre de la CASA pour se diffuser dans tous les Alpes-Maritimes et le département voisin du Var. Cela fait réfléchir sur la pertinence de l'échelle territoriale prise pour avoir une réflexion sur l'emploi d'autant plus que la logique de décentralisation des universités et des instituts de recherche publique s'est inversée avec la mise en place des pôles de compétitivité.

On comprend à travers ces quelques orientations du projet d'agglomération de la CASA qu'une grande partie des problèmes que rencontraient le technopôle de Sophia-Antipolis, et qu'il ne pouvait pas résoudre dans la mesure où il se situait sur différentes communes, sont désormais envisagés à une nouvelle échelle instituée par l'État. Il l'a jugé plus pertinente comme l'attestent les études de la préfecture, de la DATAR et de différents bureaux d'études qui ont montré que l'agglomération serait mieux à même de résoudre les problèmes d'environnement, d'habitat, de transport, etc. Des incertitudes demeurent pourtant. Les carences du technopôle semblent plus nécessiter une prise en compte de périmètres à géométrie variable permettant des actions plus ciblées qu'une intégration dans un périmètre imposé par l'État. Le territoire de l'agglomération ne semble pas adéquat pour résoudre des problèmes tels que celui de la congestion des transports. Il faut réfléchir à l'instauration d'une inter-intercommunalité à envisager pour certaines thématiques et notamment celle-ci dans la mesure où une grande partie des actifs du parc réside dans les bassins de vie périphériques (en particulier cannois et grassois). On peut ainsi penser que l'intercommunalité généralisée à la "Chevènement » est un leurre, certaines thématiques convenant tout à fait au cadre classique d'un syndicat intercommunal qui pouvait s'y consacrer pleinement en n'ayant qu'un seul objectif.

\section{Conclusion}

Si l'idéal de la création d'une ville de la science et de la sagesse a porté Sophia-Antipolis, il a débouché sur l'instauration d'un des technopôles européens les plus dynamiques. Trente-cinq après son lancement, Sophia-Antipolis est une réussite qui possède les défauts de ses qualités : inflation foncière, engorgement des transports, manque de lisibilité des relations entre entreprises. Les acteurs publics, gestionnaires du technopôle, qui ont joué un rôle fondamental dans sa création, semblant se satisfaire de leur succès, n'ont pas su prévenir et dépasser ces déséconomies qui sont devenues structurelles. Devant ce constat, les acteurs privés se sont regroupés pour faire entendre leur point de vue et proposer des solutions. La création d'une nouvelle échelle d'intervention décidée par l'État, une communauté d'agglomération, est alors apparue comme un moyen d'envisager des problèmes (logement, transport) à une échelle plus appropriée. Mais en y regardant de plus près, les mésententes entre acteurs publics ont été exacerbées et n'ont généré qu'un statu quo préjudiciable à la gouvernance du technopôle. Les enjeux mêmes du parc interrogent également l'ambition de la communauté d'agglomération en particulier en ce qui concerne ses échelles d'intervention. La question de la pertinence des nouveaux groupements intercommunaux qui devaient regrouper en leur sein un ensemble de thématiques leur permettant d'avoir une vision et une action plus globales est posée. Cela est d'autant plus inquiétant que le rôle des acteurs publics apparaît fondamental dans sa capacité à encourager l'ancrage territorial des entreprises de haute technologie, élément déterminant dans la concurrence sans pitié que se livrent les régions à l'heure de la globalisation. 


\section{Bibliographie}

Benko (G.), 1991. - Géographie des technopôles, Masson, Paris, 215 p.

Camagni (R.), Maillat (D.), 2006. - Milieux Innovateurs. Théories et politiques, édition Economica, Anthropos, Paris, 502 p.

CERDA (I.), 1979. - La théorie générale de l'urbanisation, Paris, Seuil, 247 p.

Chamboredon (J.-C.), Lemaine (M.), 1970. - « Proximité spatiale et distance sociale, les grands ensembles et leur peuplement ", Revue française de sociologie, $n^{\circ}$ XI-1, p. 3-33.

FACHE (J.), 2002. - Les territoires de la haute technologie, éléments de géographie, Rennes, PUR, 158 p.

—, 1999 - «La définition des industries de la haute technologie ", Méditerranée, n 3, p. 41-48.

GafFard (J.-L.), 1993. - «Innovation et territoires en Europe », dans SAVY (M.), Veltz (P.) (dir.), Les nouveaux espaces de l'entreprises, Marseille, DATAR/L'Aube, 199 p.

Grondeau (A.), 2000. - Lois d'Intercommunalité : implications et hypothèses d'applications sur la communauté d'agglomération Antibes-Sophia-Antipolis, Mémoire de Maîtrise, Département Géographie et Aménagement, Université de Nice-Sophia-Antipolis, 148 p.

—, 2001. - Études du logement sur Sophia-Antipolis, Mémoire de Master, Faculté de Droit, Université de Nice-Sophia-Antipolis, 35 p.

—, 2003. - Éléments de localisation et de développement des zones technopolitaines, l'exemple de Sophia-Antipolis, Mémoire de DEA, Laboratoire de Géographie Urbaine de Paris 10, 108 p.

—, 2004. - «Entre crises et mutations urbaines, quel avenir pour les technopôles? », Villes en Parallèle, Document 3 : De la Ville outil à la ville ludique, p. 47-56.

—, 2005. - Le modèle technopolitain en question : l'exemple comparé de la Silicon Valley et de SophiaAntipolis, Les cahiers nantais de la Géographie, n 62-63, p. 89-97.

Howard (E.), 1969. - Les cités-jardins de demain, Paris, Dunod (traduction), 125 p.

Le Corbusier, 1943 (rééd., 1957). - La Chartes d'Athènes des CIAM, Paris, Minuit, 190 p.

MumFord (L.), 1964. - La cité à travers l'histoire, Paris, Seuil (traduction), 777 p.

Querré (M.), 2002. - « Territoire et gouvernance locale : le cas de Sophia-Antipolis », Géographie, Économie, Société, vol. 4, n² 2, p. 225-246.

SaXenian (A.), 1994. - Regional Advantage, Culture and Competition, California, Berkeley, Université de Californie in Silicon Valley and Route 128/Harvard University Press, 226 p.

Sсотт (A.), 1999. - « Les bases géographiques de la performance industrielle », Géographie, Économie, Société, vol. 1, n² 2, p. 259-280.

Wackermann (G.), 1992. - «Les pôles technologiques, une mode ou une nécessité », La documentation française, $141 \mathrm{p}$.

Cet article a été reçu le 20 janvier 2006 et définitivement accepté le 5 septembre 2006. 